\title{
Rapid Impedance
}

\section{Spectrum Measurements}

for State-of-Health

\section{Assessment of Energy Storage Devices}

\section{SAE 2012 World Congress \& Exhibition}

The INL is a

U.S. Department of Energy

National Laboratory

operated by

Battelle Energy Alliance

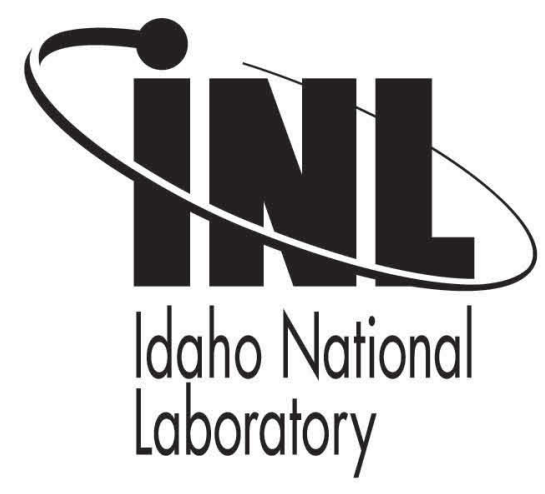

\author{
Jon P. Christophersen \\ John Morrison \\ William Morrison \\ Chester Motloch
}

April 2012

This is a preprint of a paper intended for publication in a journal or proceedings. Since changes may be made before publication, this preprint should not be cited or reproduced without permission of the author. This document was prepared as an account of work sponsored by an agency of the United States Government. Neither the United States Government nor any agency thereof, or any of their employees, makes any warranty, expressed or implied, or assumes any legal liability or responsibility for any third party's use, or the results of such use, of any information, apparatus, product or process disclosed in this report, or represents that its use by such third party would not infringe privately owned rights. The views expressed in this paper are not necessarily those of the United States Government or the sponsoring agency. 


\title{
Rapid Impedance Spectrum Measurements for State-of-Health Assessment of Energy Storage Devices
}

\author{
Jon P. Christophersen \\ Idaho National Laboratory \\ John Morrison \\ Montana Tech of Univ of Montana \\ William Morrison \\ Qualtech Systems Inc \\ Chester Motloch \\ Motloch Consulting, Inc.
}

\begin{abstract}
Harmonic Compensated Synchronous Detection (HCSD) is a technique that can be used to measure wideband impedance spectra within seconds based on an input sum-of-sines signal having a frequency spread separated by harmonics. The battery (or other energy storage device) is excited with a sum-of-sines current signal that has a duration of at least one period of the lowest frequency. The voltage response is then captured and synchronously detected at each frequency of interest to determine the impedance spectra. This technique was successfully simulated using a simplified battery model and then verified with commercially available Sanyo lithium-ion cells. Simulations revealed the presence of a start-up transient effect when only one period of the lowest frequency is included in the excitation signal. This transient effect appears to only influence the low-frequency impedance measurements and can be reduced when a longer input signal is used. Furthermore, lithium-ion cell testing has indicated that the transient effect does not seem to impact the charge transfer resistance in the mid-frequency region. The degradation rates for the charge transfer resistance measured from the HCSD technique were very similar to the changes observed from standardized impedance spectroscopy methods. Results from these studies, therefore, indicate that HCSD is a viable, rapid alternative approach to acquiring impedance spectra.
\end{abstract}

KEY WORDS: impedance spectra, rapid measurements, state-of-health, lithium-ion

CITATION: Christophersen, J., Morrison, J., Morrison, W. and Motloch, C., "Rapid Impedance Spectrum Measurements for State-of-Health Assessment of Energy Storage Devices," SAE Int. J. Passeng. Cars - Electron. Electr. Syst. 5(1):2012, doi:

10.4271/2012-01-0657.

\section{INTRODUCTION}

Battery technologies and other energy storage devices (e.g., fuel cells and ultracapacitors) have become critical components to several industries including automotive, military, space, medical, electric utility, and consumer electronics. Since battery systems can be very expensive, successful manufacturing and implementation in the field benefit from accurate, in-situ monitoring techniques for stateof-health and remaining useful life assessment. State-ofhealth $(\mathrm{SOH})$ is a measure of the battery's condition relative to its rated condition at beginning of life; remaining useful life (RUL) is an estimation of the remaining battery usage given its previous history and present power and energy demand. Developing industry standards for $\mathrm{SOH}$ and RUL, however, has been challenging because of the complexity of the problem. Passive monitoring techniques (e.g., observing changes in voltage and current along with ambient temperature) can yield valuable information about battery behavior, but the acquired data generally only track changes in capacity and state-of-charge (SOC), which is the percentage of remaining capacity relative to the rated 
capacity $[\underline{1}, \underline{2}, \underline{3}, \underline{4}]$. With increasing demands on the battery performance, however, simple passive monitoring is becoming an insufficient measure of $\mathrm{SOH}$. Knowledge of resistance rise, and the corresponding power fade, is also critical for a complete assessment of battery health. Resistance is typically determined using a volt/ohm meter at a specific measurement frequency or with active pulse tests over an SOC range using a constant current signal and observing the voltage response $[\underline{5}, \underline{6}, \underline{7}, \underline{8}]$, but these approaches are not well suited for rapid battery diagnostics (or other energy storage devices).

Recently, AC impedance has been emphasized as a useful measure of battery SOH and RUL $[\underline{9}, \underline{10}, \underline{11}, \underline{12}, \underline{13}, \underline{14}, \underline{15}, \underline{16}]$. Impedance spectra are acquired through low-level, chargeneutral input signals at various frequencies, and the data have been shown to correlate well with the corresponding resistance and power capability results that are determined from standardized pulse tests [9]. However, full-spectrum electrochemical impedance spectroscopy (EIS) typically requires expensive and delicate equipment operating in a laboratory environment. EIS measurements also tend to be sequential and can take a long time to complete (usually from ten minutes to an hour depending on settings), especially when very low frequencies are included [10]. As a result, most of the research to date has emphasized either offline EIS measurements or online measurements at only a small set of frequencies (e.g., sequentially measuring the impedance at a low, medium, and high frequency) to assess battery health and life $[\underline{12}, \underline{13}, \underline{14}, \underline{15}, \underline{16}]$.

\section{BACKGROUND}

\section{ELECTROCHEMICAL IMPEDANCE SPECTROSCOPY}

Electrochemical impedance spectroscopy testing generally consists of a small, charge-neutral AC voltage signal applied to the battery over a broad range of frequencies (e.g., $10 \mathrm{kHz}$ to $10 \mathrm{mHz}$ ). The current response is then measured to determine the impedance at each desired frequency. Alternatively, an AC current signal is injected into the battery and the voltage response is measured. A typical EIS Nyquist curve measured at $60 \%$ SOC for a commercially available Sanyo SA cell $(1.2 \mathrm{Ah})$ is shown in Figure 1. The point at which the high-frequency tail crosses the real axis is the ohmic resistance $\left(R_{O}\right)$ within the battery, which includes the effects from the electrolyte, electrodes, tabs, etc. The semicircle in the mid-frequency region identifies the charge transfer resistance $\left(R_{C T}\right)$. The charge transfer resistance is primarily influenced by the kinetic reactions at the electrodes due to an imposed constant voltage or current signal. The low-frequency tail on the right is known as the Warburg impedance that is caused by the diffusion of ions [17].

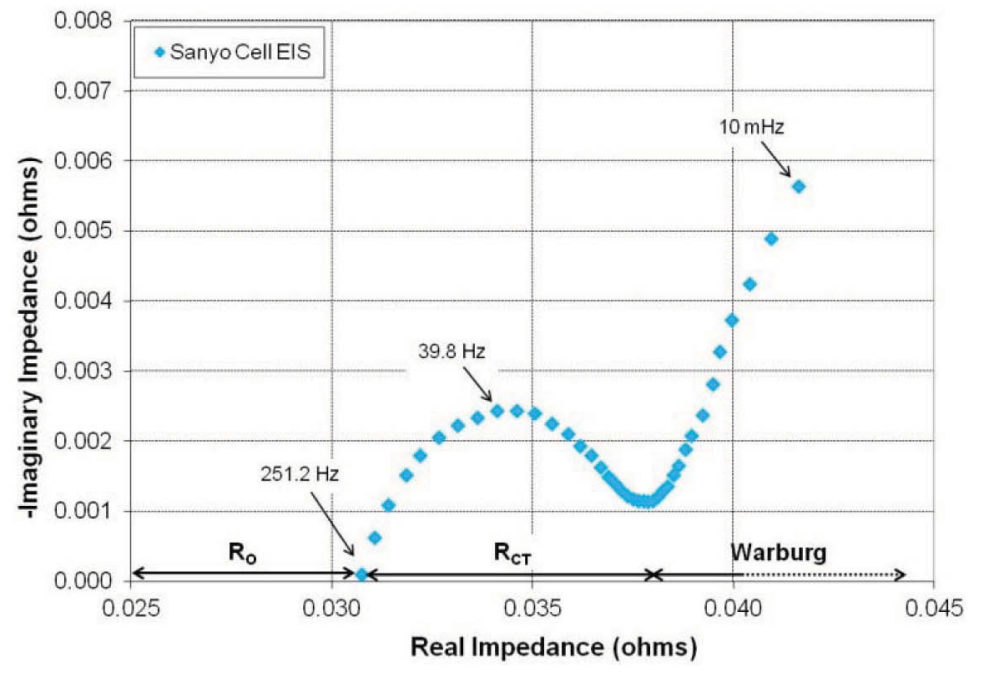

Figure 1. EIS Nyquist curve description.

Figure 2 shows EIS measurements for a Sanyo SA lithium-ion cell at different stages of life. It was subjected to more than 80,000 shallow cycles (i.e., approximately $1 \%$ $\triangle \mathrm{SOC}$ swings) at a test temperature of $50^{\circ} \mathrm{C}$, as described below. There is a small increase in the ohmic resistance, approximately $3 \mathrm{~m} \Omega$, which may come from the solid electrolyte interphase layer and other side reactions consuming lithium from the electrolyte as the cell is aged [18]. The majority of the impedance growth, however, occurs in the mid-frequency charge transfer resistance, where the semicircle grows in both height and width as a function of cell age; the growth in charge transfer resistance is approximately $6 \mathrm{~m} \Omega$. The low-frequency Warburg tail shifts to the right due to the increased cell resistance but generally retains the same slope and is otherwise unaffected by cell aging.

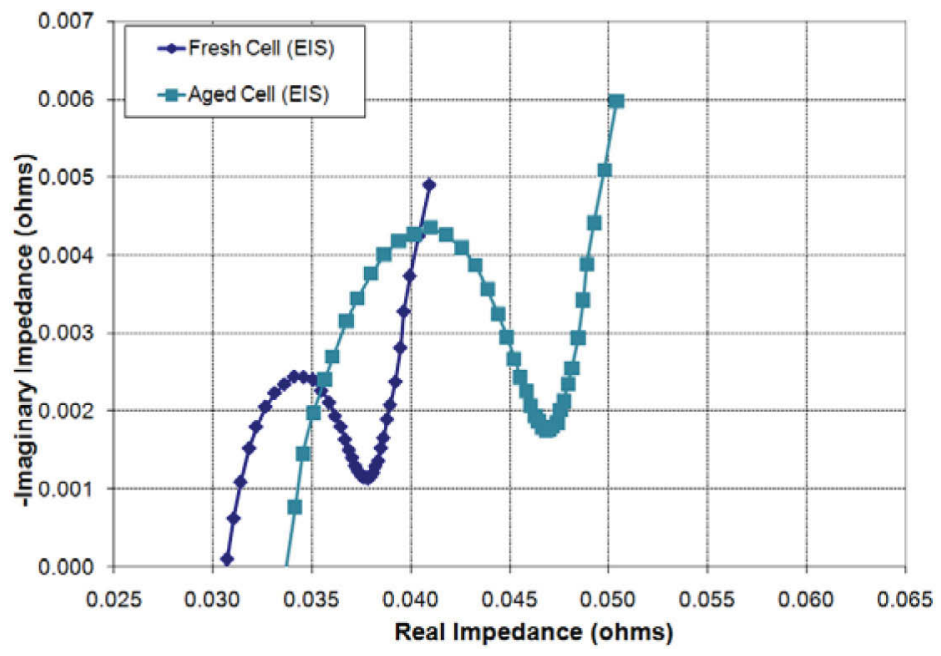

Figure 2. EIS Nyquist curves for an aging Sanyo SA cell. 
The change in measured impedance as a function of aging has been shown to linearly correlate with the resistance growth and power fade that are determined from standardized pulse tests $[\underline{9}-\underline{10}]$. These observations have indicated that impedance measurements can be used as a benign alternative for SOH and RUL assessment [10-11]. Typical EIS measurements, however, must be performed offline in a laboratory setting, or online at a very small set of sequentially measured frequencies, and this is not practical for embedded, near real-time monitoring. Rapid, broadband impedance measurement techniques, including Compensated Synchronous Detection (CSD), have recently been developed for online, in-situ applications as a means of monitoring changes in resistance and power as the battery is being operated.

\section{COMPENSATED SYNCHRONOUS DETECTION}

The CSD technique [19] consists of simultaneously exciting the battery with a set of charge-neutral current sinusoids at different frequencies that are separated logarithmically. This sum-of-sines signal, therefore, contains multiple frequencies spread over the specified range of interest. For accurate detection with minimal error, it is advantageous to include several periods of each discrete frequency. The minimum number of periods required for an accurate measurement depends on the number of frequencies included in the overall excitation signal. Studies have shown that a minimum of three periods of the lowest frequency is generally sufficient for this technique when about 12 frequencies are included in the input signal (e.g., a low starting frequency of $0.1 \mathrm{~Hz}$, having a period of 10 seconds, would require a 30-second measurement) [19-20]. If, however there are additional noise sources that that affect the response signal, or if more frequencies or interest are included in the excitation signal, more periods of the lowest frequency will be required to minimize the crosstalk interference.

The impedance magnitude and phase can be synchronously detected at each frequency within the sum-ofsines signal using the input current and measured voltage response. Since the duration of the measurement is not infinite, the input and response signals appear as sinc functions in the frequency domain. These sinc functions can overlap and cause crosstalk error in the impedance spectra. To minimize this error, the initial results are compensated by first reassembling all of the components except for the frequency of interest back into a sum-of-sines signal in the time domain. This reassembled time record is then subtracted from the original response signal and synchronously detected again with significantly reduced crosstalk error. This process is repeated for each frequency component to yield more accurate impedance spectra that are directly comparable to the EIS $[\underline{19}, \underline{20}, \underline{21}]$.
The advantage of CSD is that it is a simpler implementation that can be significantly faster compared to EIS. Additionally, the technique can be successfully implemented on a hardware platform that can be designed as an embedded system. However, it is still not very well suited to near real-time, onboard applications since it requires at least three periods of the lowest frequency to successfully resolve the impedance spectra.

\section{HARMONIC COMPENSATED SYNCHRONOUS DETECTION}

A variant of CSD is Harmonic Compensated Synchronous Detection (HCSD). The HCSD technique enables the detection of impedance magnitude and phase assuming a bandwidth-limited sum-of-sines input current signal with a harmonic frequency spread. With harmonic separation, the crosstalk error observed with the CSD approach is eliminated. This removes the need for compensation and enables a measurement to be completed within only one period of the lowest frequency. The derivation of the HCSD technique is based on the input sum-of-sines signal shown in Equation (1), where $A_{i}, \omega_{i}$, and $\phi i n_{i}$ are the amplitude, radian frequency, and phase, respectively, of the $i^{\text {th }}$ input sinusoid, and $M$ is the number of sinusoids within the excitation signal [19-20]. For a logarithmic distribution, the radian frequency $\left(\omega_{i}\right)$ can be governed by Equation (2), where the $i^{t h}$ sinusoidal signal is an octave harmonic of the lowest frequency $\left(\omega_{1}\right)$. This input signal is injected into the battery, and the measured response is sampled as shown in Equation (3), where $B_{i}$ is amplitude of the $i^{t h}$ output sinusoid, $\Delta t$ is the sampling time step, oout $_{i}$ is the phase response of the $i^{\text {th }}$ output sinusoid, and $N$ is the number of points in the captured time record $[\underline{19}, \underline{20}, \underline{21}]$.

$$
x(t)=\sum_{i=1}^{M} A_{i} \sin \left(\omega_{i} t+\phi i n_{i}\right)
$$

$$
\omega_{i}=\omega_{1} \cdot 2^{i-1} \quad i=1: M
$$

$$
y[j]=\sum_{i=1}^{M} B_{i} \sin \left(\omega_{i}(j-1) \Delta t+\phi o u t_{i}\right) ; \quad j=1: N
$$




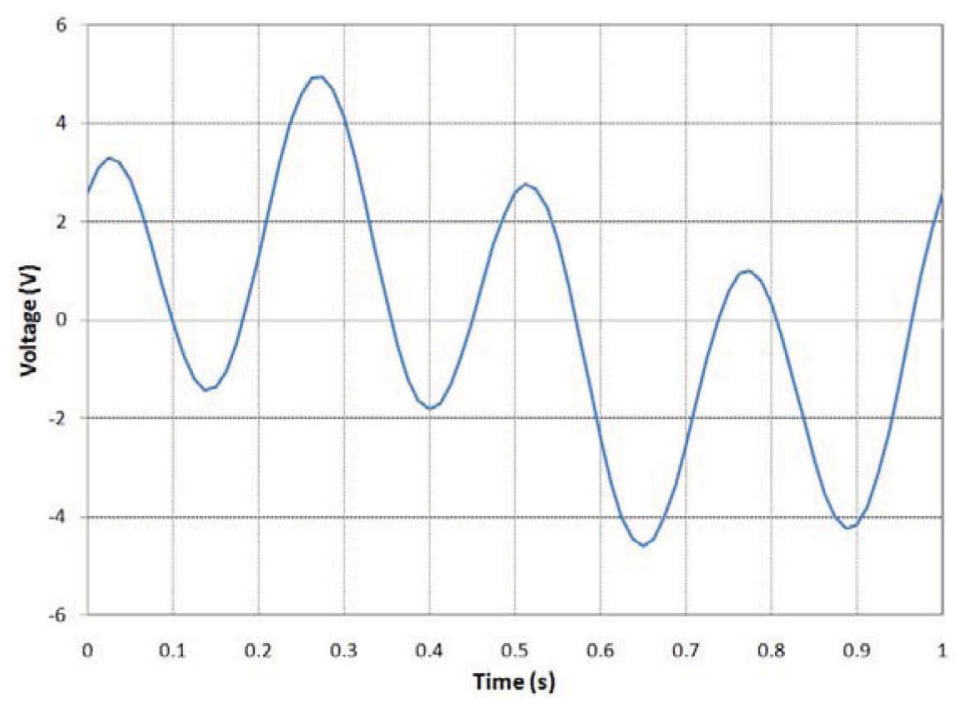

Figure 3. Sum-of-sines voltage response for one period from Equation (6).

As an example, consider a sum-of-sines input current signal, $x(t)$, consisting of two frequencies, $\omega_{1}$ and $a \omega_{1}$, respectively (where $a$ is an even integer) as shown in Equation (4). The resulting voltage response, $y[j]$, is shown in Equation (5), where the sine waves have been affected by unknown phase shifts, oout $_{1}$ and $\phi_{\text {out }}$. A sample voltage response, represented by Equation (6), for one period of the lowest frequency is shown graphically in Figure 3 , where $B=$ $[2,3], \omega_{i}=[2 \pi, 8 \pi]$ with $a=4$ (i.e., $a \omega_{1}=4.2 \pi$ ), and oout $_{i}=$ $[0, \pi / 3]$.

$$
x(t)=A_{1} \sin \left(\omega_{1} t+\phi i n_{1}\right)+A_{2} \sin \left(a \omega_{1} t+\phi i n_{2}\right)
$$

$$
\begin{aligned}
y[j]= & B_{1} \sin \left(\omega_{1}(j-1) \Delta t+\phi o u t_{1}\right)+ \\
& B_{2} \sin \left(a \omega_{1}(j-1) \Delta t+\phi o u t_{2}\right) ; \quad j=1: N
\end{aligned}
$$

$$
\begin{aligned}
y[j] & =2 \sin (2 \pi(j-1) \Delta t)+3 \sin (8 \pi(j-1) \Delta t+\pi / 3) ; \\
j & =1: N
\end{aligned}
$$

The voltage magnitude of this signal in the frequency domain, $|Y(\omega)|$, is shown in Figure 4. The resulting sinc functions are centered at the frequencies of $\pm \omega_{0}$ and $\pm a \omega_{0}$ (i.e., \pm 1 and $\pm 4 \mathrm{~Hz}$ since $\omega_{1}=1 \mathrm{rad}$ and $a=4$ ), as expected. Furthermore, crosstalk interference from the other sinc functions converge to zero at \pm 1 and $\pm 4 \mathrm{~Hz}$ since the two input sinusoidal waves in Equation (6) are separated by an integer step factor.

This voltage response signal can then be synchronously detected for the in-phase and quadrature components. The inphase response, $S_{P}$, at the $k^{t h}$ radian frequency $\left(\omega_{k}\right)$ is shown in Equation (7). This reduces to the real component of the complex impedance as shown in Equation (8), where $\phi_{\text {detected }}$ is the detected phase for the $k^{\text {th }}$ frequency of interest. Similarly, the quadrature component, $S_{Q}$, is shown in Equation (9), and it can be reduced to the imaginary component of the complex impedance as shown in Equation (10).

$$
\begin{aligned}
& S_{P_{q}}=\frac{1}{N} \sum_{j=1}^{N} \\
& \left\{\begin{array}{l}
\frac{A_{k} B_{k}}{2}\left[\begin{array}{l}
\cos \left(\phi_{i n}-\phi \text { out }_{k}\right) \\
-\cos \left(2 \omega_{k}(j-1) \Delta t+\phi \sin _{k}+\phi o u t_{k}\right)
\end{array}\right]+ \\
\sum_{i \neq k=1}^{M} \frac{A_{k} B_{i}}{2}\left[\begin{array}{l}
\cos \left(\left(\omega_{k}-\omega_{i}\right)(j-1) \Delta t+\phi \operatorname{in}_{k}-\phi o u t_{i}\right) \\
-\cos \left(\left(\omega_{k}+\omega_{i}\right)(j-1) \Delta t+\text { in }_{k}+\phi o u t_{i}\right)
\end{array}\right]
\end{array}\right\}
\end{aligned}
$$

$$
S_{P_{k}}=\frac{A_{k} B_{k}}{2} \cos \left(\phi_{\text {detected }_{k}}\right)
$$

$$
\begin{aligned}
& S_{Q_{k}}=\frac{1}{N} \sum_{j=1}^{N}
\end{aligned}
$$

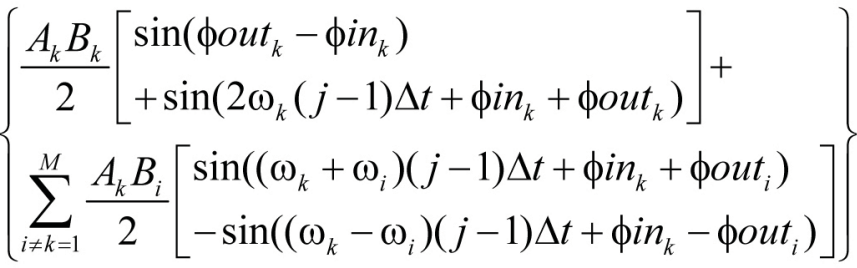




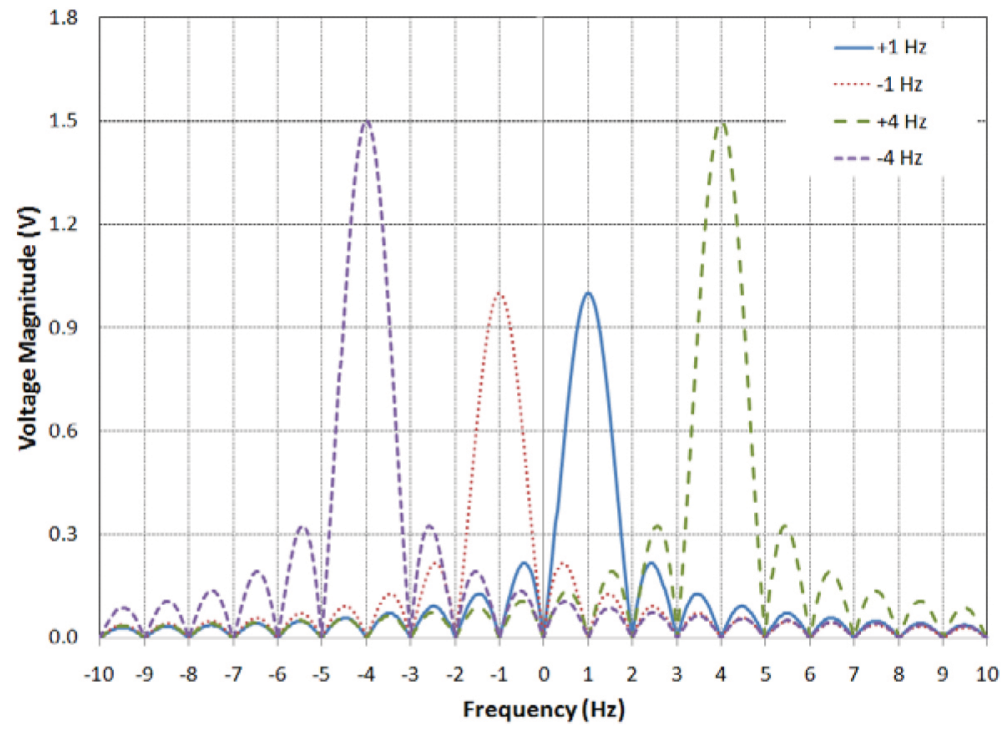

Figure 4. Magnitude response for the sinc functions in Equation (6).

$$
S_{Q_{k}}=\frac{A_{k} B_{k}}{2} \sin \left(\phi_{\text {detected }_{k}}\right)
$$

Using the example signal in Figure 3 and assuming a unity input current amplitude (i.e., $A=[1,1]$ ), the in-phase response is $S_{P}=[1,0.75]$ and the quadrature response is $S_{Q}=$ $[0,1.3]$. The magnitude and phase response of the detected impedance from Equations (8) and (10) at each frequency of interest can then be converted to the corresponding magnitude and phase response using Equations (11) and (12). From the example signal, $S_{M A G}=[2,3]$ and $S_{P H S}=[0 \pi / 3]$, which corresponds to the input values in Equation (5), as expected.

$$
\begin{gathered}
S_{M A G}=\sqrt{\left(2 S_{Q}\right)^{2}+\left(2 S_{P}\right)^{2}} \\
S_{P H S}=\tan ^{-1}\left(\frac{S_{Q}}{S_{P}}\right)
\end{gathered}
$$

This example HCSD derivation assumed a simplified sum-of-sines signal with only two frequencies added together (i.e., $M=2$ ). For the actual HCSD technique, multiple sinusoids of equal amplitudes are summed together in the excitation current signal. Figure 5 shows a simulated input current signal ( 0.5 A RMS) that consists of a sum-of-sines with octave frequencies between 0.1 and $409.6 \mathrm{~Hz}$ (i.e., $M=$ 13) for one period of the lowest frequency. For reference, the input current at the fundamental frequency $(0.1 \mathrm{~Hz})$ is included in this figure as well. The method of synchronous detection for each frequency of interest in this signal is the same as described in the example above. Since crosstalk interference is eliminated with octave harmonics, there is no need to compensate the synchronously detected magnitude and phase, as is typically done with a generic CSD analysis [20-21].

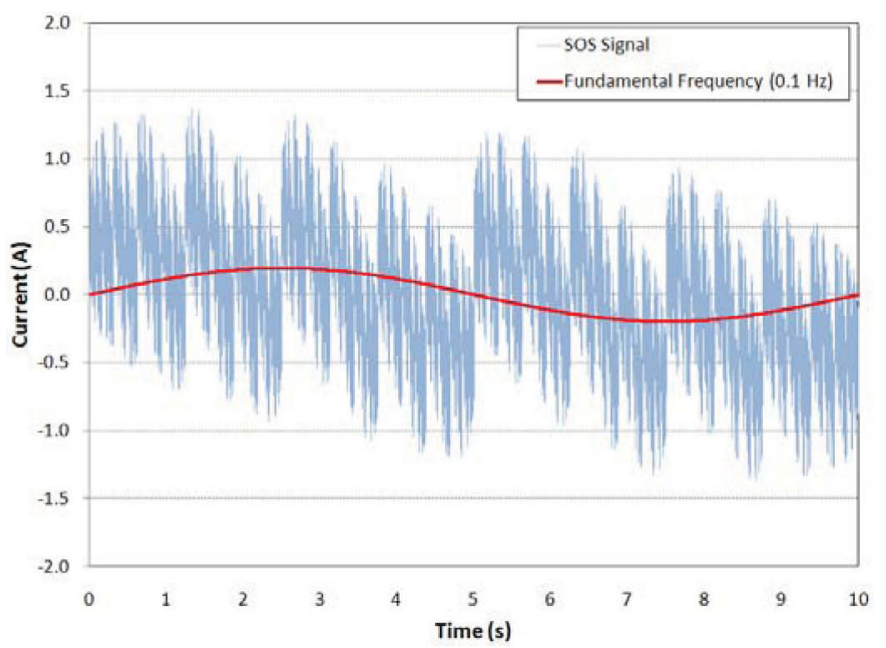

Figure 5. Simulated input sum-of-sines current signal.

The advantage of HCSD is that the impedance spectrum can be measured within one period of the lowest frequency. This technique is, therefore, much better suited for near realtime, onboard assessments of SOH and RUL. A disadvantage of HCSD is that the harmonic spread yields a lower resolution spectrum compared to the EIS methodology, especially at higher frequencies. However, as shown below, the resolution from HCSD is still capable of capturing sufficient data for assessing cell degradation. 


\section{EXPERIMENTAL}

The HCSD technique provides an alternative approach of acquiring broadband impedance measurements using hardware that could be embedded as an onboard diagnostic tool. It acquires impedance spectra rapidly using a low-level input current sum-of-sines signal. The purpose of the studies described herein was to assess the viability of HCSD measurements as an alternative to the EIS methodology. HCSD impedance measurements were first simulated based on a simplified battery model and then verified with a small set of commercially available Sanyo SA lithium-ion cells.

\section{SIMULATIONS}

The equivalent circuit for a simplified battery model is shown in Figure 6. This model was used by the Department of Energy's Applied Battery Research Program to build a test cell circuit (TCC) as a means of verifying the repeatability of EIS measurements between three national laboratories having different equipment [10]. It consists of a resistor $\left(R_{1}\right)$ in series with a parallel combination of a polarization resistance $\left(R_{2}\right)$ and capacitor $\left(C_{l}\right)$. The capacitor is coupled with an equivalent series resistance $\left(R_{3}\right)$, which is a lumped parameter describing any resistive elements in the capacitor such as current collectors, electrical leads, and contacts [22]. The polarization RC-network simulates the semicircle loop caused by the charge transfer resistance, and the ohmic resistor shifts the semicircle on the real axis of the Nyquist curve [17]. HCSD simulations consisted of applying the sumof-sines signal shown in Figure 5 as the TCC load current $\left(I_{L}\right)$ and recursively solving for the load voltage $\left(V_{L}\right)$ response. Impedance spectra were determined by synchronous detection of the simulated load voltage response.

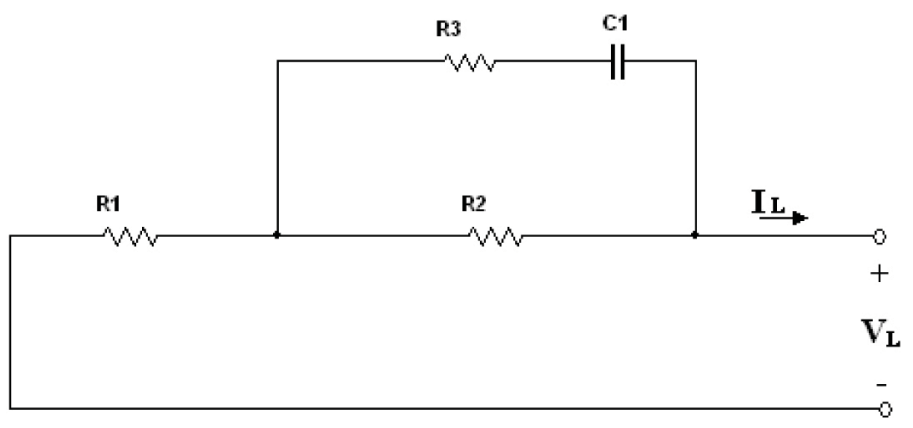

Figure 6. Test cell circuit.

\section{TESTING}

The HCSD validation testing was conducted with three high-power Sanyo SA lithium-ion cells that were 18650-size (i.e., $18 \mathrm{~mm}$ diameter and $65 \mathrm{~mm}$ length), with a rated capacity of $1.2 \mathrm{Ah}$ and a voltage range of 4.2 to $2.7 \mathrm{~V}$. The cells were placed in Tenney Junior temperature chambers that maintained ambient conditions to within $\pm 3^{\circ} \mathrm{C}$ of the target. Cell aging was conducted with a Maccor series 4000 battery tester with maximum voltage and current ratings of $10 \mathrm{~V}$ and 12.5 A, respectively. Electrochemical impedance spectroscopy measurements were conducted with a Solartron Model 1287A potentiostat/galvanostat coupled with a Solartron Model 1260A frequency response analyzer.

The three Sanyo SA cells (labeled as Cell A, B, and C) were continuously cycled at $50^{\circ} \mathrm{C}$ using the charge-sustaining cycle-life profile shown in Figure 7 [23]. This profile was established for full-size battery packs but was scaled by a sizing factor of 1400 to accommodate the power ratings for the Sanyo SA cells (e.g., the $27 \mathrm{~kW}$ discharge power pulse was scaled down to $19.3 \mathrm{~W}$ ). An elevated test temperature $\left(50^{\circ} \mathrm{C}\right)$ was used to accelerate cell degradation and yield measureable changes in performance during aging. Reference performance tests (RPTs) were conducted at $30^{\circ} \mathrm{C}$ every two weeks (i.e., every 13,440 cycles) to periodically gauge cell degradation. As part of the RPT, the cells were subjected to both EIS and HCSD measurements at the open-circuit voltage corresponding to $60 \%$ SOC (i.e., $3.89 \mathrm{~V}$ ). The EIS measurements covered a frequency range of $10 \mathrm{kHz}$ to $0.1 \mathrm{~Hz}$ with ten points per decade of frequency. The HCSD measurements ranged between $1638.4 \mathrm{~Hz}$ to $0.1 \mathrm{~Hz}$ in an octave harmonic spread; impedance data at frequencies greater than $1638.4 \mathrm{~Hz}$ could not be performed since the prototype hardware used an anti-aliasing filter that had a cutoff frequency of $2 \mathrm{kHz}$. This did not impact the results, however, since measurements at higher frequencies tend to capture instrumentation artifacts instead of cell behavior $[\underline{9}$, 10].

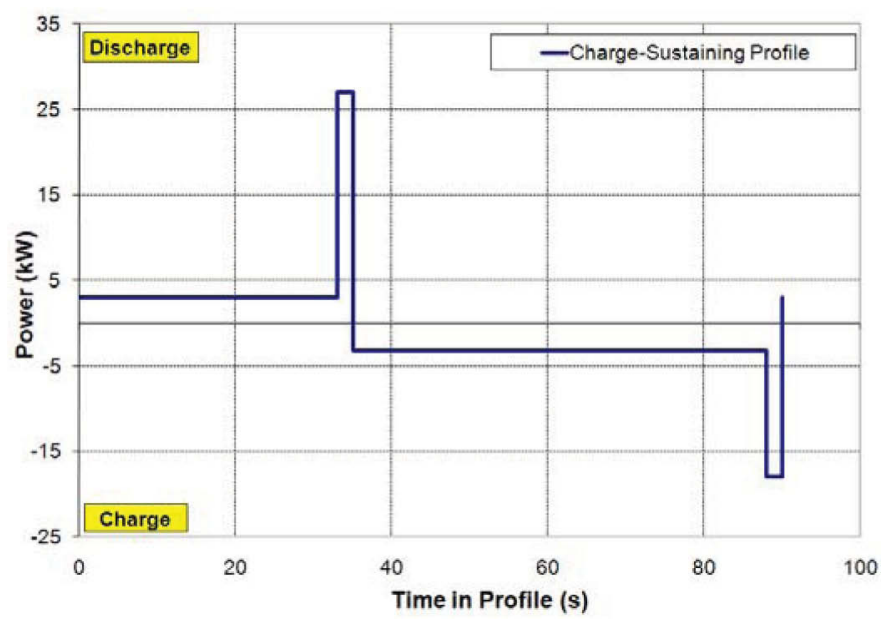

Figure 7. Charge-sustaining cycle life test profile [23].

\section{RESULTS AND DISCUSSION}

\section{SIMULATION RESULTS}

The theoretical equivalent impedance of the TCC (Figure 6) is shown in Equation (13) and the circuit parameter values used for simulations are shown in Equation (14). These parameter values correspond to the actual test cell circuit that was built for EIS validation [10]. The theoretical response at 
each frequency of interest between 0.1 and $409.6 \mathrm{~Hz}$, inclusive, is shown in Figure 8 with solid circles. At very high frequencies, the capacitor behaves as a short circuit, so $R_{1}$ is in series with a parallel combination of $R_{2}$ and $R_{3}$, resulting in an ohmic resistance of $12.5 \mathrm{~m} \Omega$. At very low frequencies, the capacitor behaves as an open circuit, and the TCC impedance becomes the sum of $R_{1}$ and $R_{2}$, or $15 \mathrm{~m} \Omega$. Thus, the charge transfer resistance for the TCC, which corresponds to the width of the semicircle, is $2.5 \mathrm{~m} \Omega$.

$$
\begin{gathered}
Z=R_{1}+\frac{R_{2}+s C_{1} R_{2} R_{3}}{1+s C_{1}\left(R_{2}+R_{3}\right)} \\
R_{1}=10 \mathrm{~m} \Omega \\
R_{2}=5 \mathrm{~m} \Omega \\
R_{3}=5 \mathrm{~m} \Omega \\
C_{1}=21 \mathrm{~F}
\end{gathered}
$$

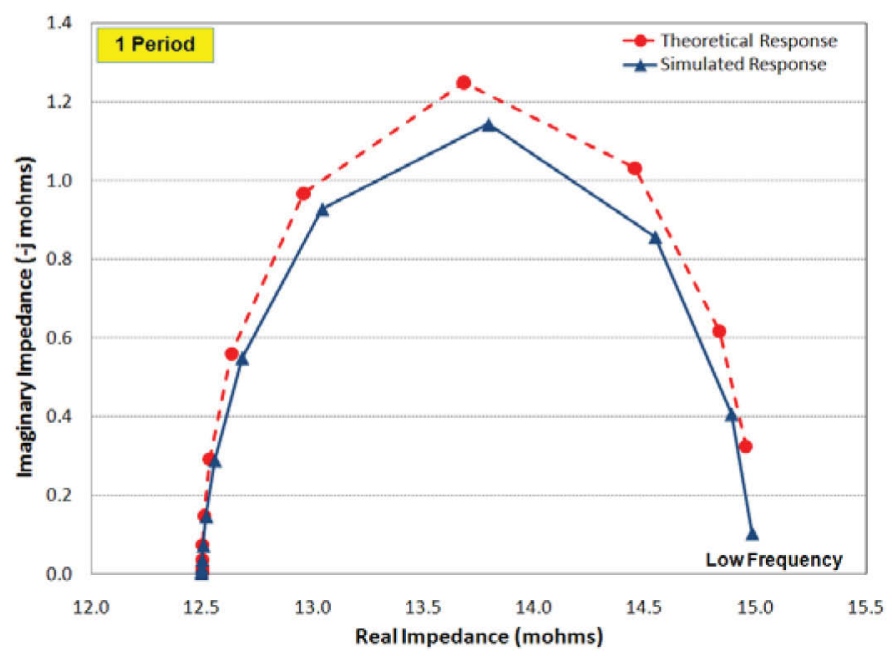

Figure 8. TCC response with one period of the lowest frequency.

The response of the TCC based on the HCSD technique using the input sum-of-sines signal given in Figure 5 is also shown in Figure 8 with solid triangles. The simulated response matches the theoretical behavior at high frequencies, but tends to deviate at lower frequencies. This deviation, however, is reduced when more periods of the lowest frequency are included in the input sum-of-sines signal. Figure 9 shows the detected impedance spectra for an input load current that has (a) five and (b) ten periods of the lowest frequency compared to the theoretical response of the TCC. This translated into a sum-of-sines signal that was 50 and 100 seconds long, respectively. As the duration of the load current input signal increased, the accuracy of the detected impedance at lower frequencies improved in both the real and imaginary components.

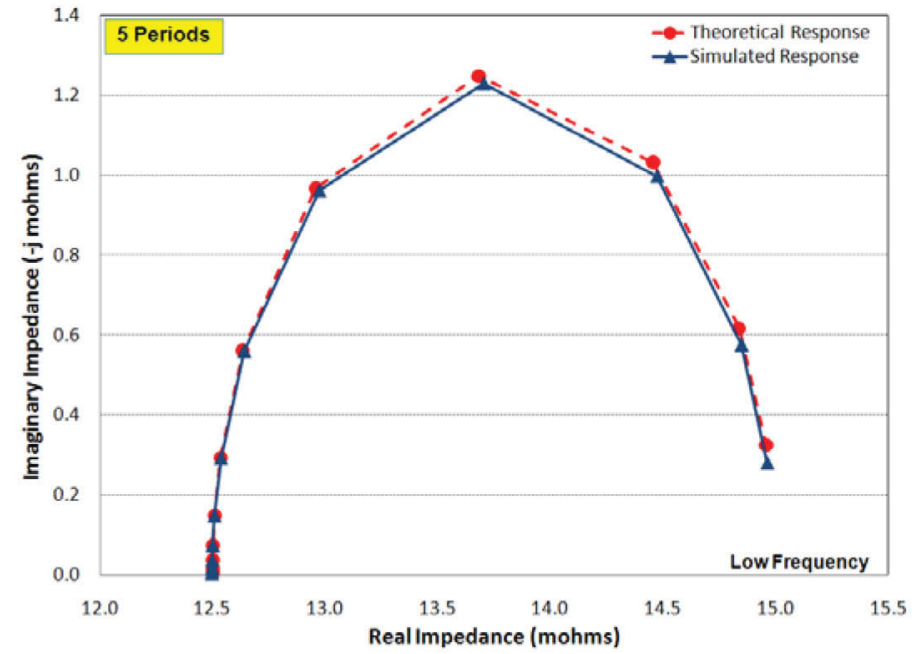

Figure 9a. TCC response with five periods of the lowest frequency.

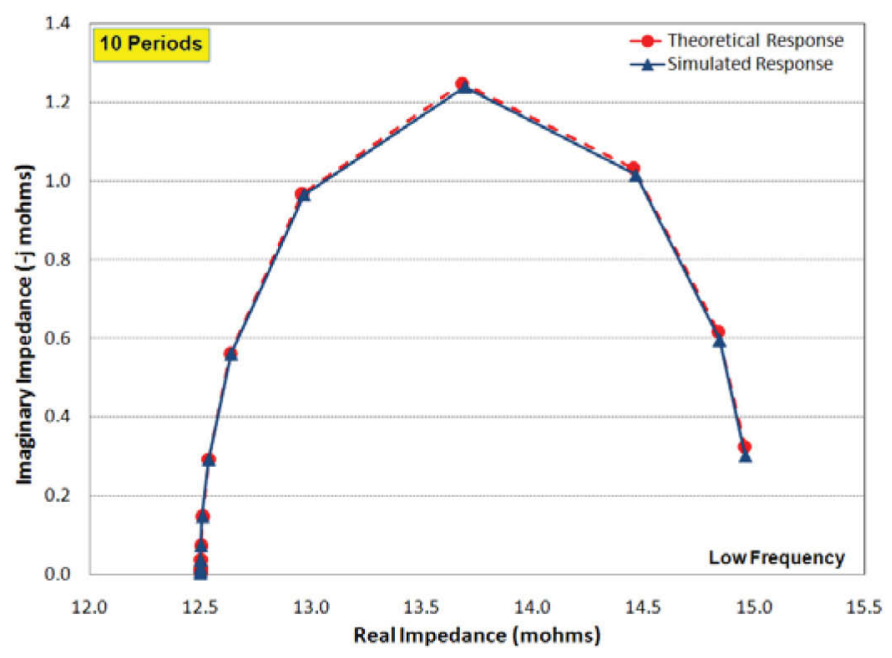

Figure 9b. TCC response with ten periods of the lowest frequency.

These simulation results imply the presence of a start-up transient effect due to the size of the capacitor [20]. At higher frequencies, the synchronously detected sinusoidal signal within the sum-of-sines contains multiple periods, so the voltage response is able to reach steady state. As the frequency of the detected sinusoidal signal decreases, however, fewer periods are included within the sum-of-sines, and the impedance is determined before a full steady-state condition can be achieved, which introduces error into results. When the duration of the sum-of-sines signal is increased, the voltage response is capable of achieving steady-state conditions at lower frequencies, which yields improved impedance spectra compared to the theoretical response. Despite these transient behaviors, however, simulations based on the TCC model demonstrate that the 
HCSD technique is capable of yielding an accurate impedance spectrum based on a sum-of-sines signal.

\section{CELL TESTING}

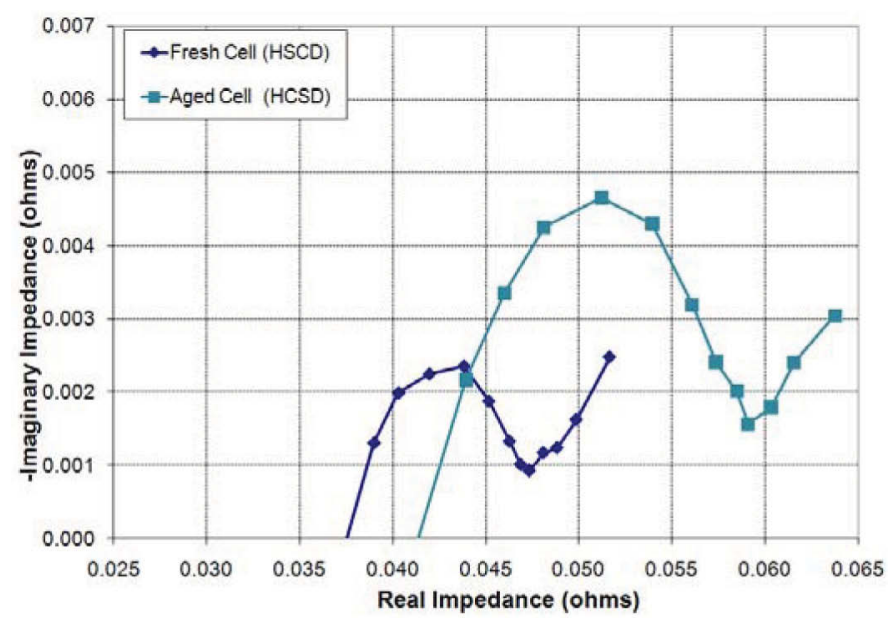

Figure 10a. HCSD Nyquist curves for an aging Sanyo SA cell.

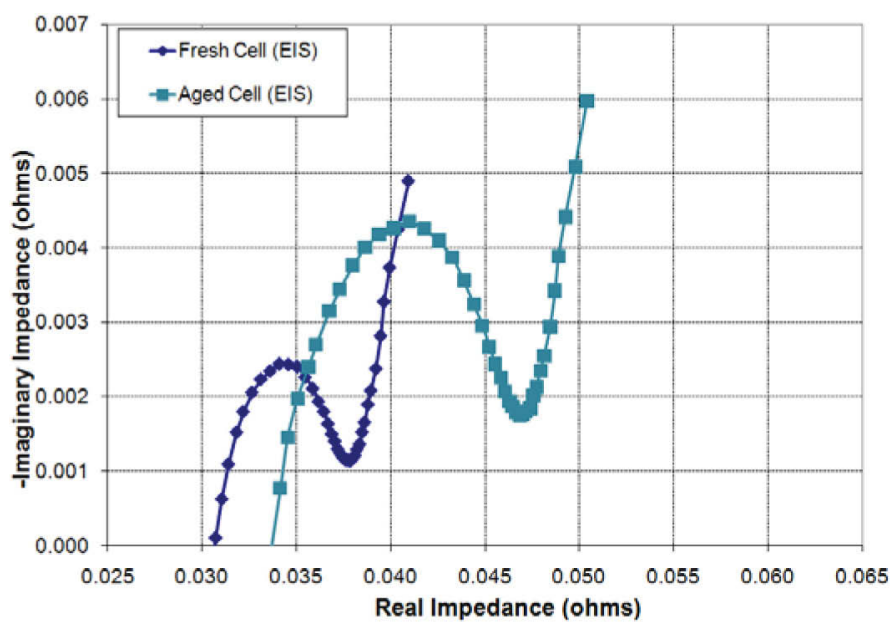

Figure 10b. EIS Nyquist curves for an aging Sanyo $S A$ cell.

The three Sanyo cells used for this study were cycled for a total of 12 weeks (i.e., 80,640 cycles) at $50^{\circ} \mathrm{C}$. Figure $10 \mathrm{a}$ shows HCSD measurements at beginning and end of life for a representative cell. The ohmic resistance, $R_{O}$, grew approximately $4 \mathrm{~m} \Omega$, while the majority of the impedance growth occurs in the mid-frequency charge transfer resistance (approximately $8 \mathrm{~m} \Omega$ ), which is consistent with the corresponding EIS results (Figure 2, shown again in Figure $10 \mathrm{~b}$ for convenience). As expected, there are fewer data points available from the HCSD impedance spectra compared to EIS because of the octave harmonic separation. The HCSD spectra (Figure 10a) also have slightly larger ohmic resistances compared to the EIS results (Figure 10a). This implies that the contact resistance between the cell and the prototype HCSD hardware is slightly higher than with the EIS equipment.

Figure 11 shows both the EIS and HCSD impedance spectra at each RPT for a representative cell (Cell A). The HCSD results in each case were first normalized to the corresponding EIS spectra at the ohmic resistance, and then the spectra pairs were artificially shifted on the real axis (i.e., the abscissa) to more clearly compare the results. The tensecond HCSD measurements show very similar behavior compared to the EIS spectra, which took approximately ten minutes to complete. The low-frequency Warburg tail from the HCSD impedance spectra, however, shows some deviation compared to EIS at each RPT. The source for this difference could be a combination of calibration errors and electrochemical transient effects. Phase errors may be present in the spectra since current shunts were used to calibrate the system on the real axis [21], but calibration of the imaginary component has not yet been developed. Improved calibration techniques that include the phase are presently under investigation

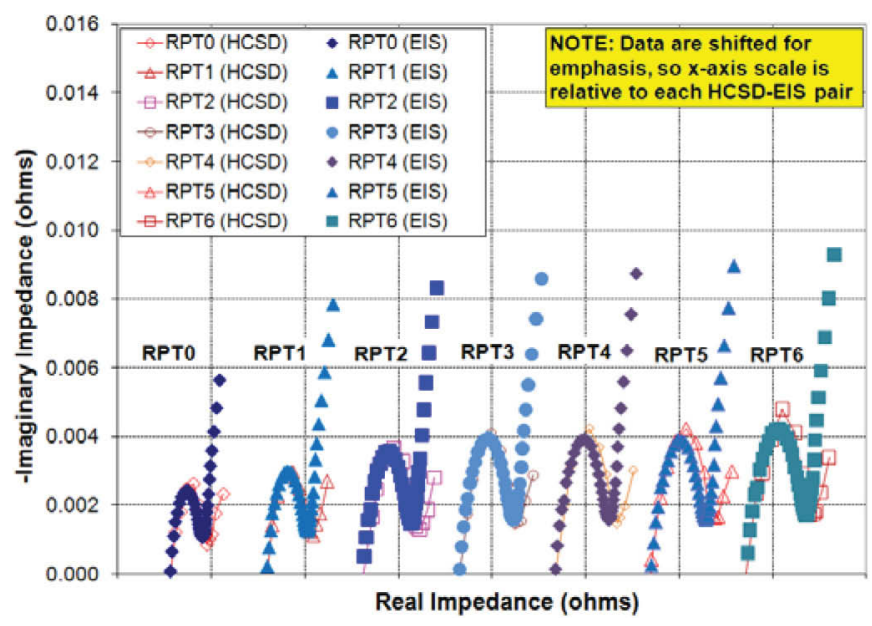

Figure 11. EIS and HCSD spectra for a representative cell through RPT6.

Furthermore, as with the simulation results, transient effects may be present due to the smaller number of periods that are included within the input sum-of-sines signal at lower frequencies, but this transient behavior can also be diminished if longer input signals are implemented. Figure 12 shows HCSD measurements for a Sanyo SA cell at $60 \%$ SOC (i.e., $3.89 \mathrm{~V}$ ) and $30^{\circ} \mathrm{C}$ after one, five, and ten periods of the lowest frequency. This translated to an input sum-of-sines signal with durations of ten, fifty, and one hundred seconds, respectively, based on a starting frequency of $0.1 \mathrm{~Hz}$. The angle of the Warburg tail increased and more closely resembled EIS results when more periods were included in the sum-of-sines excitation signal. This implies that the diffusion rate does not reach steady state within one period of the lowest frequency. The EIS results do not show this transient effect since the measurements are sequential and begin with high frequencies (i.e., the diffusion rate has 
reached steady state at the time the Warburg tail is measured). The rate of diffusion, however, does not seem to affect the width of the charge transfer resistance, since the location of the semicircle trough is similar in all cases.

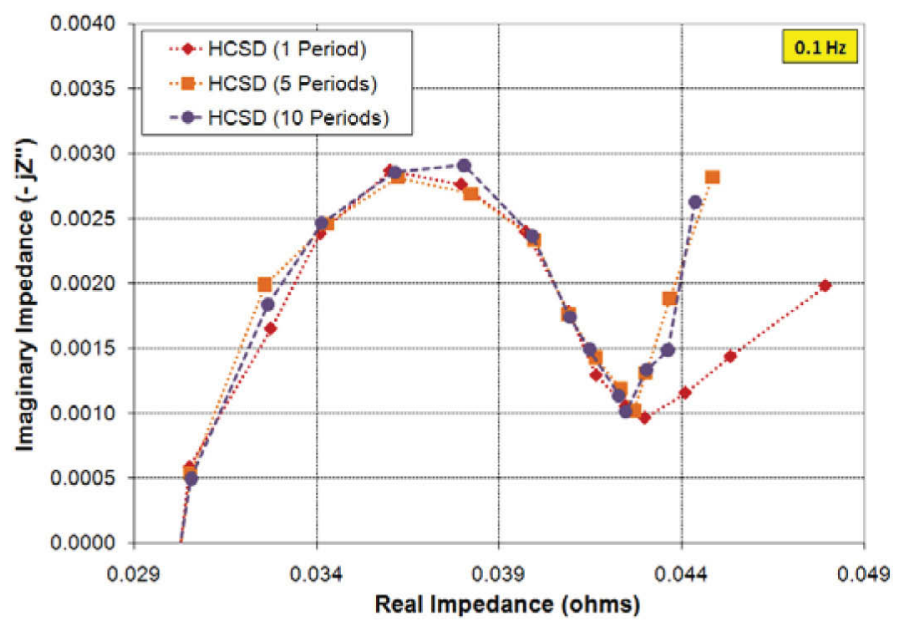

Figure 12. HCSD response with one, five, and ten periods of the lowest frequency.

Despite deviations in the Warburg tail, the HCSD impedance spectra were still capable of capturing the growth in charge transfer resistance in the mid-frequency region. Since the low-frequency tail obscures the full detail of the mid-frequency region, the growth in charge transfer resistance over time can be approximated by the change in the real impedance at the low-frequency trough of the semicircle with respect to the initial (fresh) data [ $\underline{9}-\underline{10}]$. This methodology also has the advantage of capturing the small growth in ohmic resistance as well. Figure 13 shows the measured HCSD real impedance at the semicircle trough plotted against the corresponding EIS measurement, and Table 1 provides the slope, intercept, and correlation coefficient $\left(\mathrm{r}^{2}\right)$ values for the linear regression fits. All three cells show strong correlations between EIS and HCSD, but the slope (approximately 1.25) indicates that the sum total of the ohmic and charge transfer resistances measured from the HCSD is increasing at a slightly faster rate than the corresponding EIS curve; the larger semicircle widths for HCSD can also be observed in Figure 11. One possible source for this difference could be in the test methodology. The EIS spectra were determined from a constant potential and varying current, whereas the HCSD spectra were measured based on a constant current signal and varying voltage.
Table 1. Comparisons between EIS and HCSD real impedance.

\begin{tabular}{|l|l|l|c|}
\hline Cell & \multicolumn{1}{|c|}{ Slope } & $\begin{array}{c}\text { Intercept } \\
(\mathrm{m} \Omega)\end{array}$ & $\mathbf{r}^{2}$ \\
\hline A & 1.25 & 0.25 & 0.977 \\
\hline B & 1.34 & -3.40 & 0.963 \\
\hline C & 1.24 & 0.69 & 0.997 \\
\hline
\end{tabular}

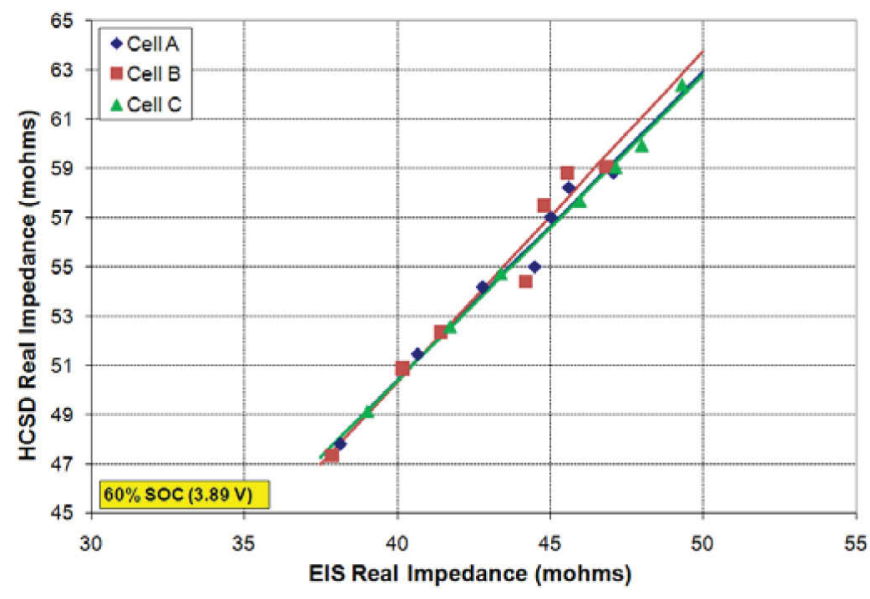

Figure 13. HCSD real impedance measurements at the semicircle trough versus the corresponding EIS results.

Nevertheless, the measured degradation rates from HCSD are essentially the same as the corresponding EIS results. Figure 14 shows the percent-growth in real impedance at the semicircle trough for the HCSD results as a function of the EIS measurements normalized to the beginning-of-life (i.e., fresh) value. A summary of the slopes, intercepts, and correlation coefficients are provided in Table 2. The intercepts for the linear regression fits were forced to be zero since the degradation of a fresh cell is, by definition, $0 \%$. The slopes are approximately unity in all cases and the results remain strongly correlated. These data indicate that, despite the larger semicircle, HCSD measurements can successfully capture the growth of the charge transfer resistance as a function of cell age.

Table 2. Comparisons between EIS and HCSD real impedance growth.

\begin{tabular}{|l|l|l|c|}
\hline Cell & Slope & $\begin{array}{c}\text { Intercept } \\
(\%)\end{array}$ & $\mathbf{r}^{2}$ \\
\hline $\mathrm{A}$ & 1.03 & 0 & 0.995 \\
\hline $\mathrm{B}$ & 1.09 & 0 & 0.990 \\
\hline $\mathrm{C}$ & 0.99 & 0 & 0.999 \\
\hline
\end{tabular}




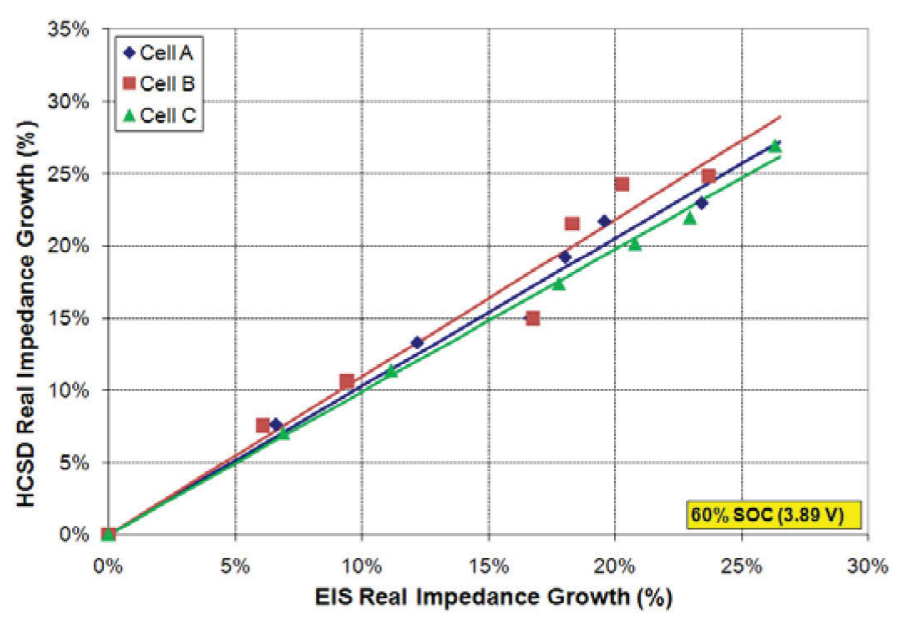

Figure 14. HCSD real impedance growth at the semicircle trough versus the corresponding EIS results.

\section{SUMMARY AND CONCLUSIONS}

The purpose of this study was to assess the viability of the HCSD measurement technique as an alternative approach to acquiring impedance spectra. Simulations based on an equivalent circuit model indicated that a sum-of-sines signal with a frequency spread separated by octave harmonics can be used to resolve the impedance, but there is also a start-up transient effect that primarily influences the results at lower frequencies. Since fewer periods were included within the sum-of-sines signal at lower frequencies, the voltage response was unable to fully reach steady-state prior to synchronous detection. Similar results were also observed with battery testing. Three Sanyo SA cells were aged at $50^{\circ} \mathrm{C}$ for more than 80,000 cycles with periodic HCSD measurements every two weeks. Although the low-frequency tail seemed to be affected by transients, the HCSD impedance spectra were very similar to the standardized EIS method in the critical mid-frequency range and showed the same degradation rates in charge transfer resistance as a function of aging. Therefore, HCSD is a rapid measurement approach that provides comparable results to standardized impedance spectroscopy methods and appears to be a viable alternative technique.

\section{ACKNOWLEDGEMENTS}

This work was prepared as an account of work sponsored by an agency of the United States Government under U.S. Department of Energy Contract No. DE-AC07-05ID14517. Funding for this work was provided by the U.S. Department of Energy Office of Vehicle Technologies. Accordingly, the U.S. Government retains and the publisher, by accepting the article for presentation, acknowledges that the U.S. Government retains a nonexclusive, paid-up, irrevocable, worldwide license to publish or reproduce the published form of this manuscript, or allow others to do so, for U.S. Government purposes. The authors gratefully acknowledge
Chinh D. Ho for ensuring that the acquired test data were of high quality.

\section{REFERENCES}

1. Sternad, M., Cifrain, M., Watzenig, D., Brasseur, G., and Winter, M.. Condition monitoring of lithium-ion batteries for electric and hybrid electric vehicles. 2009, Elektrotech. Informationstechnik, Vol. 126 (5), pp. 186-193.

2. Kurle, W.D., Johnson, S.B., Nordness, R.W., Firman, S.L., Gustavson, D.M., Choi, P.Y.. Smart Battery With Maintenance and Testing Functions. U.S. Patent 6,072,299 June 6, 2000

3. Gould, C.R., Bingham, C.M., Stone, D.A., and Bentley, P.. New Battery Model and State-of-Health Determination Through Subspace Parameter Estimation and State-Observer Techniques. 2009, IEEE Trans. Veh. Technol., Vol. 58 (8), pp. 3905-3916.

4. Okoshi, T., Yamada, K., Hirasawa, T. and Emori, A.. Battery condition monitoring (BCM) technologies about lead-acid batteries. 2006, J. Power Sources, Vol. 158, pp. 874-878.

5. Pop, V., Bergveld, H.J., Notten, P.H.L., and Regtien, P.P.L.. State-ofthe-art of battery state-of-charge determination. 2005, Meas. Sci. Tech., Vol. 16 (12), pp. R93-R110.

6. Tinnemeyer, J. Fuzzy Logic Method and Apparatus for Battery State of Health Determination. U.S. Patent 7,072,871 B1 July 4, 2006.

7. Banaei, A., Khoobroo, A., Fahimi, B.. Online detection of terminal voltage in Li-ion batteries via battery impulse response. 2009. Proceedings from the 5th IEEE Vehicle Power and Propulsion Conference. pp. 194-198.

8. Hariprakash, B., Martha, S.K., Jaikumar, A., Shukla, A.K.. On-line monitoring of lead-acid batteries by galvanostatic non-destructive technique. 2004, J. Power Sources, Vol. 137 (1), pp. 128-133.

9. Christophersen, J.P., Glenn, D.F., Motloch, C.G., Wright, R.B., Ho, C.D., Battaglia, V.S.. Electrochemical Impedance Spectroscopy Testing on the Advanced Technology Development Program Lithium-Ion Cells. 2002, IEEE Trans. Veh. Technol., Vol. 56 (3), pp. 1851-1855.

10. Christophersen, J.P., Bloom, I., Thomas, E.V., Gering, K.L., Henriksen, G.L., Battaglia, V.S., and Howell, D.. Advanced Technology Development Program for Lithium-Ion Batteries: Gen 2 Performance Evaluation Final Report, INL/EXT-05-00913. 2006.

11. Christophersen, J.P., Ho, C.D., Motloch, C.G., Howell, D., and Hess, H. Effects of Reference Performance Testing during Aging Using Commercial Lithium-Ion Cells. 2006, J. Electrochem Soc., Vol. 153, pp. A1406-A1416.

12. Kozlowski, J.D.. A Novel Online Measurement Technique for $A C$ Impedance of Batteries and Other Electrochemical Systems. 2001. Proceedings from the 16th Annual Battery Conference. pp. 257-262.

13. Tinnemeyer, J. D.L.. Multiple Model Systems and Methods for Testing Electrochemical Impedance Systems. U.S. Patent 6,778,913 B2 August 17, 2004.

14. Singh, P., Vinjamuri, R., Wang, X.Q., Reisner, D.. Fuzzy logic modeling of EIS measurements on lithium-ion batteries. 2006, Electrochim. Acta, Vol. 51 (8-9), pp. 1673-1679.

15. Yoo, J., Park, S.. An Electrochemical Impedance Measurement Technique Employing Fourier Transform. 2000, Anal. Chem., Vol. 72, pp. 2035-2041.

16. Yoon, C.O., Barsukov, Y., Kim, J.H.. Method of and Apparatus for Measuring Battery Capacity by Impedance Spectrum Analysis. 6,208,147 B1 March 27, 2001

17. Linden, D.L., Reddy, T.B. [ed.]. Handbook of Batteries. 3. New York: McGraw Hill, 2002, pages 2.26-2.29.

18. Zhang, Y., Wang, C., and Tang, X.. Cycling degradation of an automotive LiFePO4 lithium-ion battery. 2011, J. Power, Sources, Vol. 196, pp. 1513-1520.

19. Morrison, J.L., Morrison, W.H.. Method of Detecting System Function by Measuring Frequency Response. U.S. Patent 7,395,163 B1 July 1, 2008.

20. Morrison, J.L., Morrison, W.H.. Real Time Estimation of Battery Impedance. 2006. Proceedings from the IEEE Aerospace Conference.

21. Hoffmann, R.G., Slade, J.E. and Morrison, J.L.. Development and Test of a Real Time Battery Impedance Estimation System. 2006. Proceedings from the IEEE Aerospace Conference.

22. FreedomCAR Ultracapacitor Test Manual, DOE/NE-ID-11173. 2004.

23. Battery Test Manual for Plug-In Hybrid Electric Vehicles, DOE/ ID-11070. 2003 


\section{CONTACT INFORMATION}

Jon P. Christophersen, PhD.

Corresponding Author

Idaho National Laboratory

PO Box 1625

Idaho Falls, ID 83415-2209

Jon.Christophersen@inl.gov

John L. Morrison, PhD.

Montana Tech of the University of Montana

1300 W. Park

Butte, MT 59701

jmorrison@mtech.edu

Chester G. Motloch, PhD.

Motloch Consulting Inc.

725 West Riverview Dr.

Idaho Falls, ID 83401

cmotloch@gmail.com

\section{William H. Morrison}

Qualtech Systems Inc.

99 East River Drive

East Hartford, CT 06108

bill@teamqsi.com

\section{DEFINITIONS/ABBREVIATIONS}

\section{CSD}

EIS

Compensated Synchronous Detection

electrochemical impedance spectroscopy

HCSD

RPT

RUL

Harmonic Compensated Synchronous Detection

reference performance test

SOH

remaining useful life

state-of-health

SOC

TCC

state-of-charge

test cell circuit 